\title{
Por qué las empresas de medios insisten en que no son empresas de medios, por qué están equivocadas y por qué es importante
}

\author{
Philip M. Napoli y Robyn Caplan ${ }^{1}$
}

\begin{abstract}
El presente artículo es una traducción ${ }^{2}$ del texto de Napoli, P y Caplan, R. (2017), Why media companies insist they're not media companies, why they're wrong, and why it matters, First Monday, 22 (5). Recuperado de https:// firstmonday.org/ojs/index.php/fm/article/view/7051/6124

Cómo citar: Napoli, P. y Caplan, R. (2019). Por qué las empresas de medios insisten en que no son empresas de medios, por qué están equivocadas y por qué es importante (Traducción de Mariela Baladrón, Ana Bizberge, Bernadette Califano, Cecilia Fariña y Guillermo Mastrini). Revista Hipertextos 12(7), pp. 15-46. DOI: https://doi.org/10.24215/23143924e002
\end{abstract}

\section{Resumen}

Una postura común entre las plataformas de redes sociales y los agregadores ${ }^{3}$ de contenido es su resistencia a ser caracterizados como empresas mediáticas. En cambio, compañías como Google, Facebook y Twitter insisten reiteradamente en que deben ser consideradas como empresas puramente tecnológicas. Este artículo critica la posición que sostiene que estas plataformas son compañías tecnológicas en lugar de empresas de medios, explora la racionalidad que subyace a esta

\footnotetext{
${ }^{1}$ Philip M. Napoli (Ph.D., Northwestern University) es profesor emérito James R. Shepley de Políticas Públicas en la Escuela de Políticas Públicas de Sanford en la Universidad de Duke, donde también es miembro afiliado al Centro DeWitt Wallace de Medios y Democracia. Sus intereses de investigación incluyen instituciones y políticas de medios. Su próximo libro se titula Media technocracy: Algorithmic media and the public interest (Columbia University Press).

Robyn Caplan (candidata a Ph.D., Rutgers University) es investigadora en el Instituto de Investigación de Datos y Sociedad de Nueva York. Sus intereses de investigación incluyen tecnología y gobierno, medios, datos y democracia, y el papel de actores públicos y privados como intermediarios de información y datos. Ha sido becaria en el GovLab de la Universidad de Nueva York, y becaria emergente Milton Wolf 2015 del Center for Global Communication Studies en la Escuela de Comunicación Annenberg de la Universidad de Pensilvania. 2 Traducción autorizada por los autores, realizada por Mariela Baladrón, Ana Bizberge, Bernadette Califano, Cecilia Fariña y Guillermo Mastrini. La edición y cuidado estilístico estuvo a cargo de Mariela Baladrón, Ana Bizberge y Bernadette Califano.

${ }^{3}$ N. del T.: En su versión original, el artículo utiliza en algunas ocasiones la palabra "agre gadores de contenidos" [content aggregators] y en otras se refiere a "curadores de contenidos" [content curators]. En esta traducción se optó en la mayoría de los casos por la noción de "agregadores de contenidos” por ser el término más utilizado en español, y porque alude de modo más cabal a la tarea de selección y organización de contenidos que realizan estas empresas.
} 
idea y tiene en cuenta las implicancias políticas, legales y de política pública asociadas con la aceptación o el rechazo de esta postura. Como ilustra este artículo, no se trata de una mera distinción semántica, ya que la precisa clasificación de los servicios y las tecnologías de comunicación a lo largo de la historia tiene profundas ramificaciones sobre cómo estas tecnologías y servicios son considerados por quienes elaboran políticas públicas y por las cortes judiciales.

Palabras clave: Medios de comunicación, Redes sociales, Empresas de tecnología, Tecnologías digitales, Políticas de comunicación.

\begin{abstract}
A common position amongst social media platforms and online content aggregators is their resistance to being characterized as media companies. Rather, companies such as Google, Facebook, and Twitter have regularly insisted that they should be thought of purely as technology companies. This paper critiques the position that these platforms are technology companies rather than media companies, explores the underlying rationales, and considers the political, legal, and policy implications associated with accepting or rejecting this position. As this paper illustrates, this is no mere semantic distinction, given the history of the precise classification of communications technologies and services having profound ramifications for how these technologies and services are considered by policy-makers and the courts.
\end{abstract}

Keywords: Mass media, Social networks, Tech companies, Digital technologies, Communication policies.

\title{
Resumo
}

Uma posição comum entre plataformas de mídia social e agregadores de conteúdo é sua resistência a serem caracterizadas como empresas de mídia. Em vez disso, empresas como Google, Facebook e Twitter insistem repetidamente em que devem ser consideradas como empresas puramente tecnológicas. Este artigo critica a posição de que essas plataformas são empresas de tecnologia em vez de empresas de mídia, explora a lógica por trás dessa idéia e leva em conta as implicações políticas, legais e de políticas públicas associadas à aceitação ou rejeição desta posição. Como ilustra este artigo, não é uma mera distinção semântica, porque o histórico da classificação precisa de serviços e tecnologias de comunicação tem ramificações profundas em como essas tecnologias e serviços são considerados por aqueles que elaboram políticas públicas e pelos tribunais judiciais.

Palavras-chave: Mídia, Redes Sociais, Empresas de tecnología, Tecnologias digitais, Politicas de comunicação. 


\section{Introducción}

En los últimos años han surgido una serie de controversias en torno de las redes sociales. Estas polémicas estallaron alrededor de diversos temas, tales como las acusaciones de sesgo en la construcción de las listas de tendencias en Facebook y Twitter (Nunez, 2016a, 2016b; Thune, 2016); las críticas a estas plataformas por censurar fotos de interés histórico/periodístico (Scott e Isaac, 2016); la evidencia de una creciente prominencia de reportes con noticias falsas en sus feeds; y las preocupaciones sobre el posible impacto de la diseminación de noticias falsas en los resultados de las elecciones de Estados Unidos y Europa (Bell, 2016; Robinson, 2016).

Esta secuencia de eventos ha centrado la atención pública en preguntar si las plataformas de redes sociales están funcionando como organizaciones de noticias y cómo lo están haciendo o de qué manera; junto con el interrogante de si deberían comportarse como organizaciones periodísticas (Isaac, 2016; Jarvis, 2016; Manjoo, 2016). Estas discusiones tienen lugar mientras los datos indican que las plataformas de redes sociales se han establecido como uno de los principales mecanismos a través de los cuales los individuos acceden y consumen noticias (Gottfried \& Shearer, 2016). La ironía potencialmente preocupante de esta situación es que estas compañías de redes sociales que desempeñan un papel cada vez más importante e influyente en el ecosistema de noticias e información insisten desde hace tiempo en que no son organizaciones de noticias, ni siquiera compañías de medios. En cambio, estas empresas reclaman que deberían ser pensadas únicamente como empresas tecnológicas (veáse, por ejemplo, Dixon, 2014; D’onofro, 2016). En este sentido, las plataformas de redes sociales siguen el patrón establecido y sostenido por otras empresas de medios digitales como Google y Apple, al insistir en que no deben ser consideradas como compañías mediáticas.

Algunos analistas han señalado esta creciente desconexión entre cómo estas compañías se perciben a sí mismas -y quieren ser percibidas por otros- y cómo realmente funcionan en el ecosistema contemporáneo de noticias e información (Caplan, 2016; Bell, 2016; Bogust, 2016; Ingram, 2012; Snip, 2016). El objetivo aquí es llevar el análisis unos pasos más allá, al indagar críticamente los fundamentos articulados y las motivaciones subyacentes de esta posición, así como considerar las implicancias más amplias de aceptar este argumento. Una meta central de este artículo es ilustrar cómo este razonamiento encaja en un 
Por qué las empresas de medios insisten en que no son empresas de medios, por qué están equivocadas y por qué es importante

plano más amplio con los esfuerzos de estas compañías de "discursivamente... encuadrar sus servicios y tecnologías" (Gillespie, 2010:348). Como señala Gillespie (2010), muchas empresas de medios digitales usan términos como "plataforma" estratégicamente, "tanto para posicionarse con el fin de alcanzar ganancias actuales y futuras como para lograr un punto más favorable en el plano regulatorio entre las protecciones legislativas que los benefician y las obligaciones que no lo hacen, y establecer un imaginario cultural dentro del cual su servicio tenga sentido" (Gillespie, 2010). Vemos así que esta auto-definición en tanto empresas de tecnología y la fuerte resistencia a cualquier clasificación como empresas mediáticas constituye una dimensión central de su marco discursivo, que tiene importantes implicancias para los marcos legales y regulatorios que se aplican a estas plataformas.

Esta crítica a los argumentos de que las plataformas de redes sociales (y agregadores de contenido digital en sentido más amplio) deben ser considerados puramente como empresas tecnológicas y no como empresas mediáticas se basa en perspectivas económicas, históricas y políticas sobre los medios de comunicación, en un esfuerzo por esclarecer los puntos significativos de continuidad, más que de distinción, entre las plataformas tradicionales y las nuevas para las noticias y la información. Al desentrañar y criticar este argumento, este artículo busca demostrar la importancia de lo que en la superficie puede parecer una distinción semántica sin consecuencias. Como ilustra este trabajo, hay implicancias legales y de política pública fundamentales, asociadas al modo en que estas compañías se definen a sí mismas y son definidas por quienes elaboran políticas públicas y por el público.

La primera sección de este artículo presenta y refuta los argumentos comúnmente postulados por aquellas compañías que buscan ser percibidas como empresas de tecnología en lugar de medios de comunicación. La segunda explora las principales razones de estas compañías para mantener esta posición. La tercera parte considera las implicancias más amplias de aceptar los argumentos cada vez más sutiles acerca de que son compañías de tecnología en lugar de empresas de medios. En las conclusiones se considera la necesidad de formular nuevas aproximaciones conceptuales que registren del mejor modo posible esta creciente intersección entre tecnología y medios de comunicación. 


\section{Por qué las compañías de medios insisten en que no son medios de comunicación y por qué están equivocadas}

Al parecer, en el entorno comercial actual de las empresas tecnológicas, estas firmas ven su identidad cada vez más siguiendo los enfoques tecnológicos que toman para sus actividades que en términos del sector particular en el que operan. Uno de los ejemplos más prominentes -y controversiales- de la actualidad involucra a Uber. Esta compañía ha sostenido firmemente que es una empresa de tecnología en lugar de una empresa de transporte porque, según un representante de la compañía, "no transportamos bienes o personas, nuestros socios sí lo hacen. Solo facilitamos eso" (Cukier, 2016: 1). Las ramificaciones de aceptar o rechazar este argumento son profundas porque si Uber es percibido como una compañía de transporte, entonces debe estar sujeto a las regulaciones bajo las cuales opera esa industria. Por otro lado, si es considerado como empresa de tecnología, entonces estas regulaciones de la industria de transporte simplemente no aplican; lo que puede representar una importante fuente de ventaja competitiva (véase, por ejemplo, Carney, 2015). Definitivamente, Uber no se encuentra solo al mantener esta postura. Las compañías automotrices, de finanzas y telecomunicaciones sostienen de forma parecida que deben ser vistas puramente como empresas tecnológicas (Napoli \& Caplan, 2016).

De modo similar, parece que una característica que define a las plataformas de redes sociales y a los agregadores de contenido digital es la consistencia con la que se han resistido a ser caracterizados como compañías mediáticas y, en cambio, han insistido en que deben ser consideradas puramente como empresas tecnológicas (véase, por ejemplo, Helft, 2008; Mickey, 2013). Incluso compañías que están inequívocamente en el negocio de producción de contenidos mediáticos, como Gawker y Vox, han tenido CEOs que sostuvieron que son compañías tecnológicas y no empresas de medios (véase, por ejemplo, Sicha, 2011; Tjaardstra, 2015).

Antes de analizar los detalles de los argumentos presentados por estas compañías, es importante reconocer que la amplia terminología de "compañía de medios" en torno a la cual se han estructurado estos argumentos es algo problemática. Los medios no son un constructo único, monolítico (a pesar de la tendencia a tratarlos así en el discurso que nosotros examinaremos). Primero, desde la perspectiva de la regulación y de la política pública, los medios 
Por qué las empresas de medios insisten en que no son empresas de medios, por qué están equivocadas y por qué es importante

electrónicos ${ }^{4}$ como la televisión y la radio (y con la imposición de las regulaciones de neutralidad de Internet, también Internet), son considerados fundamentalmente diferentes de otros medios, ya que generalmente se les otorga niveles más bajos de protección de la Primera Enmienda y operan bajo requisitos reglamentarios más explícitos para servir al interés público (Napoli, 2001). Esta es una distinción importante que generalmente no encuentra cabida en los argumentos que se discuten más abajo. Además, también está ausente la importante ( $\mathrm{y}$ a veces borrosa) distinción entre entretenimiento y medios informativos. Desde el punto de vista regulatorio y de política pública, la producción, distribución y consumo de noticias ha sido un gran punto de motivación y foco de quienes elaboran políticas (Napoli, 2015); no obstante, algunas preocupaciones sobre la dimensión cultural de los medios dedicados al entretenimiento han motivado también la regulación y elaboración de políticas (Napoli, 2008). Como será ilustrado más abajo, estos puntos de distinción con frecuencia son importantes al considerar el argumento de que las compañías tecnológicas no son empresas de medios.

Pasamos ahora a los argumentos centrales a favor de la postura que sostiene que las plataformas de redes sociales y agregadores de contenido digital son empresas de tecnología y no de medios. En cada caso, analizaremos las perspectivas económica, histórica y política sobre las industrias de medios para ilustrar las debilidades centrales de cada argumento.

\section{1. "No producimos contenido"}

El argumento principal entre las compañías de medios digitales para sostener la posición de que no son medios de comunicación es que muchas de esas empresas no producen contenido original sino que, por el contrario, son meros facilitadores de la distribución de contenido creado por sus usuarios. Algunas de las compañías que han creado este argumento, después se han integrado verticalmente en la creación de contenido, siguiendo un modelo bastante predecible en la historia de los medios, en el cual los distribuidores de contenido inevitablemente persiguen las ventajas estratégicas y económicas de ser también creadores de contenido (véase Napoli, 2016). De esta forma, por ejemplo, en

\footnotetext{
${ }^{4}$ N. del T.: la regulación en los Estados Unidos está basada en el soporte tecnológico, mientras que otros países consideran la clasificación por tipo de servicio. Por lo tanto, "medios electrónicos" [electronic media] son aquellos mediados electrónicamente para llegar a su audiencia, como la radiodifusión, los servicios de cable, satélite e internet, a diferencia de la prensa gráfica.
} 
2016 YouTube comenzó a producir series originales para su servicio de suscripción, mientras que (como será discutido más adelante en las conclusiones), Facebook ha iniciado recientemente una incursión en la creación de contenidos de video (Etherington, 2016; Perez and Shieber, 2017; Dougherty and Steel, 2015). Sin embargo, dada la persistencia pasada -y posiblemente futura- de este argumento entre los distribuidores de contenido, es importante que reciba un escrutinio detallado.

El ejecutivo de Google, Eric Schmidt (junto con otros miembros de la empresa), desplegó este argumento frecuentemente durante años, declarando: "Nosotros no hacemos nuestro propio contenido. Nosotros conseguimos el contenido de algún otro, más rápido" (en Sullivan, 2006: 1). Facebook también ha propuesto este argumento. El responsable de Soluciones Globales de Marketing de Facebook ha sostenido, "De hecho nosotros nos definimos como una compañía de tecnología... Las compañías de medios son conocidas por el contenido que ellas crean" (en Fiegerman, 2016: 1). Mark Zuckerberg, CEO de Facebook, ha mantenido esta posición durante años (véase Fiveash, 2016), incluso en noviembre de 2016 en su respuesta a las acusaciones que sostuvieron que la diseminación de noticias falsas vía Facebook podría haber afectado las elecciones presidenciales en EE.UU. (Roberts, 2016). Dick Costolo, entonces CEO de Twitter, señaló: "Pienso en nosotros como una empresa de tecnología dado que considero que el futuro de la compañía está en construir una plataforma extensible que permita a desarrolladores y empresas agregar valor a Twitter en un sentido que sea beneficioso para Twitter y para nuestros usuarios... Yo no necesito ni quiero estar en el negocio de los contenidos" (en Bilton, 2012:1). Steve Jobs, de Apple, en sus últimos años enfatizó este punto de la misma manera en el momento del lanzamiento de iTunes. Crispado por la sugerencia de un periodista de Esquire de que Apple se estaba volviendo una empresa de medios, Jobs argumentó: "Nosotros no somos una compañía de medios. Nosotros no poseemos medios. Nosotros no poseemos música. No tenemos películas o televisoras. Nosotros no somos una compañía de medios. Nosotros somos sólo Apple" (citado en Langer, 2003: 2). El tema fue tan sensible para Jobs que terminó abruptamente la entrevista en ese momento.

Este argumento obviamente invita a discutir sobre los tipos de actividades que son centrales para nuestro entendimiento sobre lo que hacen las compañías de medios. Tradicionalmente, la organización industrial de los medios de comunicación ha sido descripta fundamentalmente por tres actividades (que 
Por qué las empresas de medios insisten en que no son empresas de medios, por qué están equivocadas y por qué es importante

raramente se excluyen mutuamente): 1) producción (ejemplificada por los creadores de contenidos como los productores de noticias o los estudios de televisión); 2) distribución (el proceso de transportar el contenido desde los productores hacia los consumidores); y, 3 ) exhibición (el proceso de proveer contenido directamente a las audiencias) (véase Picard, 2011).

La digitalización y la convergencia de los medios han significado, en algunos casos, que estos procesos se fusionaran, ya que el contenido puede ser ahora distribuido directamente al usuario final. El declive de los exhibidores tradicionales como las librerías y las disquerías frente a la digitalización de contenidos y la distribución digital representa un caso ilustrativo en este sentido. Es difícil argumentar que los exhibidores tradicionales como los cines, las librerías, o las disquerías deberían ser pensados como compañías de medios. Sin embargo, los reemplazos para estas entidades, tales como Amazon, iTunes y Netflix, son fundamentalmente diferentes, ya que utilizan infraestructura de medios electrónicos para distribuir contenido a las audiencias, en una forma pura de bien público, de manera que se asemejan más a los tradicionales curadores y distribuidores de contenidos, tales como los sistemas de cable y las estaciones de radiodifusión abierta. Esto también ocurre de diferentes maneras en el caso de las plataformas de redes sociales como Twitter, Snapchat y Facebook, las que ahora sirven como medio central para la distribución de contenidos de diversos creadores, mientras que también ofrecen un punto de exhibición para los usuarios, quienes acceden al contenido a través de dichas plataformas mediante una variedad de dispositivos. Y, con servicios como Facebook Live (que permite una transmisión directa e inmediata sobre la plataforma de Facebook) y Facebook Instant Articles (a través del cual la empresa directamente aloja contenido producido por organizaciones de noticias; véase Constine, 2015), el proceso de producción, distribución y exhibición se encuentra aún más integrado.

Enfatizamos sobre este proceso evolutivo porque la distribución es una característica definitoria de los medios tanto como lo es la creación de contenidos, como una gran cantidad de académicos especializados en medios ha dejado claro (véase, por ejemplo, Curtin y otros, 2014). La creación/ propiedad de los contenidos nunca ha servido como un punto de distinción en la definición de una compañía de medios de comunicación desde la perspectiva de los encargados de regular el sector. Hay que considerar, por ejemplo, que tanto las industrias de la televisión por cable como por satélite fueron establecidas por completo sobre el fundamento de servir exclusivamente (al menos en el inicio) 
como distribuidoras de contenido. Este hecho nunca sirvió como un mecanismo para mantener a estas compañías más allá de los límites de la autoridad regulatoria de la Comisión Federal de Comunicaciones (FCC) ${ }^{5}$. En el caso de la televisión por cable, la FCC extendió su autoridad regulatoria hacia este sector en parte por considerar al servicio como complementario de la radiodifusión (United States vs. Southwestern Cable Co., 1968). El hecho de que los sistemas de cable sirvieran como un medio central para la distribución y el acceso a contenidos (de la televisión abierta) hizo que quedaran incluidos bajo la autoridad regulatoria de la FCC, lo que actuó como un mecanismo de expansión de dicha autoridad hacia el cable.

En consecuencia, las compañías de la industria del cable y del satélite quedaron sujetas a regulaciones de propiedad y algunas obligaciones de interés público, manteniendo los criterios generales de la FCC sobre la regulación de medios electrónicos (véase, por ejemplo, FCC, 2016a). Las preocupaciones fundamentales de la política de medios sobre diversidad, competencia y localismo (véase Napoli, 2001), han caracterizado la regulación de estas industrias, independientemente de si, o hasta qué punto, estas compañías están involucradas en la creación de contenidos. Incluso, aunque los mecanismos de distribución de contenidos en el ámbito digital son desde luego diferentes de los empleados por los medios tradicionales, tal como la ejecutiva de medios digitales Elizabeth Spiers preguntó: “¿Me explicará alguien cómo la distribución digital de tu contenido hace que tu compañía sea principalmente una empresa tecnológica?” (Benton, 2014: 1). Entonces, el argumento que sostiene que la creación/ propiedad de contenido separa de manera significativa a las compañías "tecnológicas" del sector de los medios refleja o bien un entendimiento naif o mal informado de los medios, o bien un intencionado esfuerzo estratégicamente motivado para redefinir los parámetros de las compañías de medios dentro del discurso comercial y de las políticas públicas. En ambos casos, las implicancias son problemáticas, como discutiremos con mayor detalle a continuación.

\section{2. "Somos científicos informáticos"}

La segunda línea de argumentación prominente formulada por las plataformas de redes sociales y los agregadores de contenidos digitales se focaliza en la naturaleza de su personal. Específicamente, los representantes de estas

\footnotetext{
${ }^{5}$ N. del T.: La Comisión Federal de Comunicaciones (FCC, por sus siglas en inglés) es el regulador de medios y telecomunicaciones de Estados Unidos.
} 
Por qué las empresas de medios insisten en que no son empresas de medios, por qué están equivocadas y por qué es importante

compañías destacan con frecuencia el entrenamiento profesional y la formación de ellos mismos y sus empleados para sostener el argumento de que son empresas tecnológicas antes que compañías de medios. Eric Schmidt (Google) enfatizó que Google es una compañía tecnológica "dado que es dirigida por tres informáticos" (Kramer, 2006: 1). De forma similar, Be Huh (CEO de Cheezburger) ha enfatizado acerca de la proporción de empleados que son desarrolladores para sostener su argumento de que se trata de una compañía tecnológica en lugar de una empresa de medios (Koetsier, 2012).

Aquí también podemos apreciar un argumento carente de lógica o fundamento histórico. Hay que considerar, por ejemplo, que en el momento de su introducción no había ninguna maravilla tecnológica más grande que la radiodifusión abierta. Representó un salto tecnológico en los medios de comunicación de tal magnitud que probablemente solo haya sido alcanzado por la introducción de internet. Consecuentemente, la radiodifusión fue el campo de los tecnólogos e ingenieros de su tiempo, dada su relativa complejidad técnica comparada con los otros medios de comunicación disponibles como los periódicos. La especialización tecnológica estaba en el corazón de las primeras compañías de radio como RCA y Marconi Company (Woolley, 2016). La tecnología satelital representó otro avance dramático que, una vez más, requirió profesionales con un alto nivel de especialización tecnológica.

Los avances tecnológicos, así como la especialización técnica asociada, han sido fundamentales para el sector de los medios de comunicación desde -por lo menos- el advenimiento de la prensa escrita. Como señala Deuze (2007), es importante que reconozcamos "el rol central que las tecnologías ejercen en el trabajo de los medios” (Deuze, 2007: 73). Argumentar que la orientación tecnológica del personal y de los ejecutivos de una empresa es un fundamento lógico para evitar que sean pensadas como medios de comunicación refleja una comprensión desinformada de su historia y evolución, o bien un esfuerzo estratégico para acotar la definición a sus parámetros tradicionales. Cualquiera sea la razón, la premisa subyacente de que la tecnología y los medios son emprendimientos separados, sostenidos sobre habilidades y formaciones profesionales completamente diferentes, no tiene fundamentos firmes en la historia de los medios. 


\section{3. "No hay intervención editorial humana"}

Un argumento menos explícito que proponen las plataformas, evidentemente vinculado al anterior, es la afirmación frecuente de que el contenido que "subyace" no se realiza mediante el juicio humano, sino mediante algoritmos y tecnologías basadas en datos, que filtran, categorizan y clasifican información que ya existe en el sistema, y refleja aquello que los usuarios quieren. Plataformas como Google y Facebook han enfatizado reiteradamente en la falta de intervención humana en sus procesos de curación de contenidos y se han mostrado reticentes a reconocer tal intervención cuando ésta ocurre (véase, por ejemplo, Trielli et al., 2016). A raíz de la controversia por las "noticias que son tendencia" (Trending News), en un informe que sostuvo que los editores "humanos" contratados por Facebook suprimían las noticias conservadoras de su lista de tendencias, la empresa procuró minimizar la importancia que la intervención editorial humana directa jugó en el funcionamiento de su plataforma (véase, por ejemplo, Fiveash, 2016; Isaac, 2016), llegando incluso a eliminar los puestos de los periodistas y editores que supervisan los módulos de "Tendencias" como consecuencia de esta polémica (Thielman, 2016).

Esta afirmación/percepción acerca de la falta de intervención editorial humana directa es, en varios sentidos, fundamental para la lógica de concebir a estas plataformas como compañías tecnológicas en lugar de medios de comunicación. En efecto, como Gillespie (2010) ha ilustrado, el término plataforma en sí mismo ha sido estratégicamente desarrollado como forma de caracterizar a estos servicios estrictamente como neutrales, facilitadores para la creación de contenido y su diseminación, guiados por la tecnología. Mark Zuckerberg, de Facebook, ha preferido enfatizar que la plataforma simplemente proporciona herramientas a los usuarios para ayudarlos a generar sus propios contenidos y curación (Fiveash, 2016), una posición que parece ignorar -o al menos caracterizar erróneamente- el papel que desempeñan los algoritmos al priorizar y filtrar contenido para los usuarios. La reivindicación de la falta de participación humana directa en la línea editorial ayuda a profundizar esta percepción de distancia y/o neutralidad en el proceso de selección de los contenidos; un modelo que se presume fundamentalmente diferente del tipo de intervención editorial directa (y humana) que ha sido una característica definitoria de las empresas de medios tradicionales. 
Por qué las empresas de medios insisten en que no son empresas de medios, por qué están equivocadas y por qué es importante

Sin embargo, simplemente por el hecho de que los mecanismos para el ejercicio editorial -como barrera de acceso- hayan cambiado, no significa que tal identidad fundamental e institucional en tanto "gatekeepers" deba ser remodelada. Los representantes de estas plataformas probablemente argumenten que la naturaleza de su interacción con los usuarios es muy diferente a la de los medios tradicionales, ya que los usuarios de las redes sociales desempeñan un papel mucho más autónomo en la determinación del contenido que reciben. Es decir, los usuarios, en colaboración con sus redes sociales, en última instancia dictaminan el contenido que consumen, con la plataforma como facilitadora neutral. Ya sea un reflejo de ingenuidad o falta de sinceridad, esta posición simplemente ya no es posible de sostener (Gillespie, en prensa).

Para empezar, los medios siempre han intentado, hasta cierto punto, dar a las audiencias exactamente lo que quieren. En este sentido, Facebook, Twitter o Google son poco diferentes de cualquier medio de prensa, de radiodifusión o de noticias digitales que busque desesperadamente descubrir qué es lo que quieren las audiencias y luego proporcionarles exactamente eso (Nadler, 2016). La verdadera diferencia es que las plataformas de medios digitales representan mecanismos más efectivos y eficientes para hacerlo, dada la cantidad, alcance y profundidad de los datos de usuarios que pueden utilizar, lo que es una función de mayor interactividad asociada con estas nuevas plataformas. La relación entre las plataformas de redes sociales y los usuarios representa el siguiente paso en lo que Napoli (2011) ha denominado "la racionalización de la comprensión de la audiencia".

Entre los sucesos recientes podemos observar el tan criticado cambio de marca de la popular compañía de medios de comunicación Tribune Publishing ${ }^{6}$ por "Tronc" (véase Satell, 2016), como un indicador de cuán permeable es el límite que separa a las compañías de tecnología de una de medios. La metamorfosis de Tribune en Tronc parece radicar en la confianza depositada en los algoritmos y herramientas automáticas para servir mejor a los intereses de la audiencia y facilitar el uso de la automatización en la producción de contenidos (Satell, 2016). El escepticismo generalizado (e incluso la burla) que ha alcanzado este cambio de marca (véase, por ejemplo, Satell, 2016), respecto de si representa algo verdaderamente transformador, ayuda a ilustrar que una mayor dependencia de

\footnotetext{
${ }^{6}$ N. del T.: En 2016 la compañía adoptó el nombre Tronc (abreviación de “Tribune online content”) y en 2018, luego de vender sus periódicos de California, retomó su nombre anterior, Tribune Publishing.
} 
la tecnología para satisfacer a los intereses de la audiencia no sirve como una distinción significativa entre compañías de tecnología y de medios.

Además, la noción de que los algoritmos operan de una manera completamente neutral y objetiva, libre de los sesgos que caracterizan la toma de decisión editorial humana directa, ha sido desacreditada. Los algoritmos, aunque automatizados, se utilizan para clasificar, filtrar y priorizar el contenido basado en los valores internos del sistema, las preferencias y acciones de los usuarios (DeVito, 2016). A esta altura, es sabido que los ingenieros y otros actores de la compañía deben tomar innumerables decisiones en el diseño y desarrollo de algoritmos. A través de dichas definiciones y relaciones, juicios subjetivos y sesgos se codifican en los sistemas. Por estas razones, la parcialidad algorítmica se ha convertido en un fenómeno ampliamente investigado y cada vez más comprendido (véase, por ejemplo, Gillespie, 2017; Kitchin, 2017; Pasquale, 2015).

A ello se suma el hecho de que, sin importar los mecanismos de barrera de entrada utilizados, las plataformas como Facebook, Google y Twitter se encuentran a sí mismas teniendo que navegar en un rango de temas de política editorial que los ubica de modo firme en sintonía con las organizaciones tradicionales de medios, tales como diarios y radiodifusores (Gillespie, en prensa). Los temas relacionados con la protección de los usuarios de contenido ofensivo, provocativo o contenido para adultos son fundamentales para el funcionamiento de estas plataformas (véase, por ejemplo, Pierson y Dave, 2016). Esta tensión fue bien ilustrada a partir de la controversia que surgió en torno a la decisión de Facebook de censurar la publicación de un periodista noruego, que mostraba la tan conocida foto de la Guerra de Vietnam, de una joven vietnamita desnuda que huía de un ataque de napalm (Scott e Isaac, 2016). En medio de la protesta, Facebook rápidamente revirtió su decisión, considerando la importancia histórica de la fotografía (Scott e Isaac, 2016). No obstante, lo que quedó en evidencia con esta medida es que las decisiones de política editorial no son tan diferentes de las tomadas por los editores de noticias tradicionales y forman parte de lo que significa ser una plataforma de medios sociales, incluso cuando la toma de decisión editorial es manejada por algoritmos, como en el caso de la foto de Vietnam.

Y, tal como ilustró la controversia en torno a la lista de tendencias de Facebook, la intervención editorial humana a menudo desempeña un papel más 
Por qué las empresas de medios insisten en que no son empresas de medios, por qué están equivocadas y por qué es importante

importante en el proceso de gestión de contenidos de lo que comúnmente se supone (Fiegerman, 2016). Snapchat emplea a un equipo de editores de alrededor de 75 personas y también utiliza a estos empleados para entrenar a los algoritmos y tecnología que, eventualmente, permitirá automatizar la selección y clasificación de noticias para sus 100 millones de usuarios diarios (Sloane, 2016). Twitter también emplea un "equipo de curación" para examinar el contenido y destacar los eventos y tendencias en su sección "Momentos" -un segmento similar a un blog en el que los editores de la empresa trabajan para combinar los tweets y las fotos publicadas en el sitio, particularmente para los eventos de noticias que son tendencias (Twitter, 2016). Las pautas que utilizan estos equipos no son diferentes de las directrices editoriales utilizadas por muchas compañías de contenidos, las que esbozan sugerencias de contenido para promover precisión y limitar sesgos, así como pautas estilísticas acerca del tipo de titulares que los miembros del equipo deben escribir y la elección de los íconos de previsualización [thumbnails].

Debería quedar claro que este escenario apunta a que estas compañías operen no solo como empresas de medios en un sentido amplio, sino más específicamente como organizaciones de noticias, dado el grado en el que participan del flujo de noticias e información a partir de decisiones editoriales y tareas de "gatekeeping".

\subsection{La centralidad de la publicidad}

Finalmente, vale la pena señalar que todos los argumentos presentados por estas plataformas colapsan debido al hecho de que la principal fuente de ingresos para la mayoría de ellas es la publicidad. Ubicarse en el negocio de proporcionar contenidos a las audiencias y vender esas audiencias a los anunciantes es una característica definitoria del sector de los medios de comunicación (Ingram, 2012; Wolff, 2012). Cuando los anunciantes evalúan la mejor manera de llegar a sus consumidores potenciales, las fortalezas y debilidades de las plataformas de medios digitales como Facebook, Twitter y Google se analizan en conjunto con las opciones que ofrecen los medios más tradicionales, como la televisión abierta, el cable, y las publicaciones impresas y en línea. Además, estas plataformas de medios digitales están demostrando ser cada vez más efectivas en desviar los ingresos de publicidad de estos otros sectores de medios (Bhattacharyya, 2016). Desde esta perspectiva, es difícil cuestionar que todas estas empresas se encuentran operando dentro del mismo sector empresarial. Y es un negocio al 
cual, como ha demostrado la historia (ver más arriba), las empresas pueden ingresar sin poseer o producir su propio contenido.

\section{Motivaciones subyacentes}

Hasta el momento, la discusión se ha centrado en las razones explicitadas por estas empresas de medios digitales para ser consideradas compañías de tecnología en lugar de compañías de medios. El siguiente paso es mirar más allá de las explicaciones que sostienen esta lógica y considerar algunas de las razones subyacentes por las cuales este argumento se sostiene de manera sistemática.

\subsection{Apelar a la comunidad de inversores}

Quizás la razón más obvia por la cual las plataformas de redes sociales y los agregadores de contenido digital prefieren ser consideradas como compañías de tecnología en lugar de compañías de medios es que esa etiqueta trae consigo un potencial de cotizaciones mucho más altas por parte de la comunidad de inversores (véase, por ejemplo, Fox, 2014). Como aclara el inversionista Chris Dixon, la clasificación tiene implicaciones significativas en ese sector. Tal como él afirma, "una de las cosas más importantes que debe hacerse en una inversión de riesgo en una etapa posterior es pensar rigurosamente en cómo son clasificadas las empresas" (en Kafka, 2014: 1). Como se ha observado con frecuencia, la comunidad de inversores generalmente ve un mayor potencial de ingresos en el sector de la tecnología que en el de los medios de comunicación (véase, por ejemplo, Bond, 2007; Roberts, 2016), y parece haber mantenido una distinción rígida entre estos dos sectores a pesar de una creciente evidencia de lo contrario. Presentarse a sí mismo como una empresa de tecnología en lugar de una compañía de medios es "lo que los capitalistas de riesgo [quieren] escuchar" (Morrisey, 2016: 1). Como ha señalado Elizabeth Spires, directiva de medios digitales, "los inversores institucionales no financian los servicios de medios" (Benton, 2014: 1).

Hasta cierto punto, este fenómeno puede reflejar una perspectiva cultivada por las trayectorias dispares de las empresas digitales líderes y pioneras, Google y Yahoo, y quizás también por los choques culturales que fueron fundamentales para la desaparición de AOL-Time Warner. Yahoo "desplazó a los ingenieros" (Foremski, 2014: 1) desde los inicios de su historia en un esfuerzo por convertirse 
Por qué las empresas de medios insisten en que no son empresas de medios, por qué están equivocadas y por qué es importante

en una compañía de medios plena. En su apogeo, Yahoo incluso consideró la compra de la cadena NBC en un intento por integrarse como medio tradicional. Estos esfuerzos por evolucionar de un motor de búsqueda o "portal" (una denominación que fue muy popular durante un breve tiempo) a una compañía de medios completa obviamente fracasaron estrepitosamente en comparación con el enfoque estratégico más tecnocrático de Google.

La fusión de AOL-Time Warner se ha convertido en un caso de estudio icónico sobre cómo la incompatibilidad de las culturas corporativas sumergió el tipo de integración de los medios tradicionales con los nuevos medios, que la mayoría de los analistas predijo como un resultado deseable -e incluso inevitablede la convergencia digital (McGrath, 2015). La principal conclusión de este desastre fue que "la gente de la tecnología" y la "gente de los medios de comunicación" no funcionaron particularmente bien juntos y, quizás lo más importante, no parecieron ser capaces de captar la idiosincrasia de los negocios del otro (McGrath, 2015). Al final, AOL fue expulsado del conglomerado y el nombre completamente borrado de la identidad de la compañía.

Los caminos recorridos por Yahoo y AOL-Time Warner probablemente hayan ayudado a reforzar la lógica de la estrategia por mantener al menos la ilusión de distancia y distinción con respecto al sector de los medios de comunicación. El resultado final, en todo caso, es que las plataformas de redes sociales y los agregadores de contenido digital se enfrentan a un fuerte incentivo para definirse de la manera que mejor resuena con las percepciones y prioridades de la comunidad de inversores, que continúa manteniendo una estricta separación o distinción artificial entre empresas de tecnología y empresas de medios.

\subsection{Las motivaciones legales y de políticas públicas}

También es importante considerar las motivaciones legales y de políticas públicas para ser considerado como una empresa de tecnología en lugar de una compañía de medios. Como se ilustró en el ejemplo anterior de Uber, la forma en que una empresa es clasificada puede tener importantes consecuencias legales y de políticas públicas. Sin duda, esto también ocurre desde hace tiempo en el sector de los medios. 
Por ejemplo, al decidir acerca de la constitucionalidad de la Ley de Decencia en las Comunicaciones ${ }^{7}$ (que aplicaba el modelo regulatorio de los medios audiovisuales a Internet), la Corte Suprema de los Estados Unidos tuvo dificultades para definir si el trato hacia el mundo online es equiparable a la telefonía, la prensa escrita o un medio audiovisual (Stein, 1997). Dada la larga tradición en los Estados Unidos de aplicar regímenes regulatorios completamente diferentes a diversas tecnologías de comunicación en función de sus características tecnológicas, el argumento de equiparar a Internet por analogía con otro sistema de comunicación -si esto fuera posible- tiene implicancias de gran alcance a nivel normativo y de políticas públicas. Más recientemente, la decisión de la FCC de imponer regulaciones de neutralidad de red a los proveedores de servicios de Internet (ISPs) se basó en la reclasificación de los proveedores de servicios de Internet como proveedores de servicios de telecomunicaciones (similar a las compañías telefónicas) en lugar de proveedores de servicios de información (similares a los servicios de alojamiento web) (U.S. Federal Communications Commission, 2015; Rinehart, 2015). La autoridad regulatoria de la FCC sobre los proveedores de servicios de telecomunicaciones es mucho mayor que su autoridad sobre los proveedores de servicios de información (una vez más, diferentes tecnologías y servicios frecuentemente operan bajo distintos modelos regulatorios); tanto es así que el régimen de neutralidad de la red impuesto por la FCC sería inadmisible si los ISPs fueran clasificados como proveedores de servicios de información (Patel, 2014). Con la FCC de Trump es inminente la revocación de estas regulaciones de neutralidad de la red que probablemente implique otra ronda de reclasificación.

Los agregadores de contenido digital, como las plataformas de redes sociales, están comenzando a confrontar con temas similares en relación a la clasificación de sus servicios. Esto quedó bien ilustrado por algunos de los desafíos legales que enfrentó Twitter. En un caso relacionado con una citación del gobierno para obtener información (incluidos los tweets) de un usuario individual, el tribunal sugirió que Twitter era análogo a "gritar por la ventana" (Nueva York v. Harris, 2012). En otra citación que recibió Twitter por la información de la dirección IP de algunos de sus usuarios (que estaban asociados con la organización Wikileaks), la corte usó una clasificación diferente, comparando a Twitter, y las direcciones

\footnotetext{
${ }^{7}$ N. del T. Esta ley fue sancionada en 1996 en los Estados Unidos e intentó regular el material pornográfico en internet. Fue declarada inconstitucional por la Corte Suprema de ese país en 1997.
} 
Por qué las empresas de medios insisten en que no son empresas de medios, por qué están equivocadas y por qué es importante

IP utilizadas para conectarse al sitio, con el uso del teléfono (Nueva York vs. Harris, 2012).

Una pregunta fundamental que subyace a estos esfuerzos de clasificación de los servicios implica si, o en qué medida, Twitter tiene la propiedad y la autoridad editorial sobre el contenido que circula en su plataforma. Al explorar las problemáticas de la clasificación de los servicios, los analistas de la industria han señalado que "ha sido... conveniente para Twitter hacerse pasar por una empresa tecnológica cuando se trata de posibles cargas legales y regulatorias" (Rana, 2012: 1). Por ejemplo, en respuesta a una orden judicial para obtener información sobre un usuario que fue arrestado durante una protesta de Occupy Wall Street, Twitter adoptó la posición legal de que no es propietario de los tweets individuales (Rana, 2012), una postura que parece operar en conflicto con las diversas formas de decisión editorial en las que la empresa se ha visto involucrada en relación al contenido de su plataforma (véase, por ejemplo, Guynn, 2016; Ingram, 2012).

La realidad es que las plataformas como Twitter tienen un margen de maniobra sustancial para participar en la decisión editorial sobre el contenido que alojan sin activar la categorización como editor y, por lo tanto, limitando las responsabilidades legales de gran alcance que atañan a esa figura. Esta es la esencia de la Sección 230 de la Ley de Telecomunicaciones de 1996. La Sección 230 proporciona una amplia protección a las plataformas tales como los motores de búsqueda, los proveedores de servicios de Internet y los sitios de redes sociales frente a la responsabilidad legal por cualquiera de los discursos de terceros que alojan, agregan o distribuyen. Desde este punto de vista, las plataformas de redes sociales y los curadores de contenido digital están protegidos de muchas de las obligaciones negativas (es decir, las obligaciones de vigilar/proteger contra la circulación de diversos tipos de discurso) asociadas con las compañías de medios; o, en palabras de la Sección 230, "proveedores de contenido de información". Una gran parte de la vigilancia de contenidos con la que se comprometen estas plataformas, es importante enfatizar, es en realidad voluntaria (bajo lo que se conoce como la "disposición del buen samaritano"), lo cual no difiere de la forma en que la industria de la televisión por cable ha restringido durante mucho tiempo el lenguaje obsceno y la desnudez en la programación que proporcionan, a pesar de estar fuera de los límites de las reglamentaciones sobre contenidos indecentes de la FCC. 
Sin embargo, en muchos sectores de la industria de los medios de comunicación también hay una larga tradición de obligaciones afirmativas (es decir, obligaciones de proporcionar ciertos tipos de discurso). Las compañías de medios electrónicos han operado históricamente bajo un conjunto único de responsabilidades sociales impuestas por el gobierno y han planteado durante mucho tiempo que estas imposiciones son pesadas y costosas. En los Estados Unidos, estas responsabilidades sociales han tomado la forma de diverso tipo de “obligaciones de interés público" (Napoli, 2015). Incluso aquellas compañías de medios electrónicos que se dedican principalmente a la distribución de contenido (como los sistemas de cable, ISPs y prestadores de servicios satelitales) han estado sujetas a obligaciones como proporcionar a los suscriptores acceso a niveles mínimos de programación pública, educativa y gubernamental; niveles mínimos de contenidos de producción local; proporcionar acceso de banda ancha a escuelas y bibliotecas, y brindar a los candidatos políticos la posibilidad de emitir anuncios en estas plataformas a tasas reducidas (U.S. Federal Communications Commission, 2016a). Por un tiempo, tales obligaciones de interés público se volvieron particularmente agresivas, como en el caso de la Doctrina de la Imparcialidad que, durante la década de 1970 y parte de la década de 1980, exigía a los emisores (dada su importante posición de cuello de botella) que proporcionen cantidades equivalentes de cobertura a las diferentes perspectivas sobre temas controvertidos de importancia pública (Ruane, 2011).

Incluso las regulaciones estructurales por motivos económicos y la supervisión gubernamental se han impuesto históricamente de forma más agresiva en el sector de los medios de comunicación que en otros rubros de la industria, debido a las preocupaciones sobre la relación entre la competencia en los mercados de los medios y el funcionamiento efectivo del "mercado de ideas" (Napoli, 2001). Una variedad de regulaciones de la propiedad persiste en el sector de los medios electrónicos, a pesar de la creciente competencia facilitada por Internet y la reducción de las barreras de entrada para ingresar a diversos mercados de medios (U.S. Federal Communications Commission, 2016b). Las fusiones en el sector de los medios electrónicos se someten a una revisión separada de "interés público" más allá del control estándar que todas las operaciones experimentan en términos de su impacto en la competencia, en el cual las preocupaciones que no tienen ninguna relación con el efecto en la competencia pueden incluirse en el proceso de aprobación, y en el que a menudo se imponen diversas condiciones orientadas al interés público para aprobar una fusión (Sallet, 2014). 
Por qué las empresas de medios insisten en que no son empresas de medios, por qué están equivocadas y por qué es importante

El punto clave en la revisión de cómo se aplica la noción de interés público en el contexto de la regulación de los medios electrónicos es ilustrar que la clasificación como compañía de medios de comunicación ha significado históricamente una supervisión gubernamental más intensiva en la forma de obligaciones afirmativas para servir al interés público, y una regulación más estricta en áreas tales como la concentración de la propiedad. Dadas estas mayores cargas regulatorias que recaen sobre el sector de los medios electrónicos, se puede observar por qué las plataformas de medios digitales trabajarían para establecer una identidad organizativa que las ubique fuera de este modelo regulatorio.

Cabe imaginar, por ejemplo, qué sucedería si Facebook tuviera que operar bajo una "Doctrina de la Imparcialidad" para su red social. De muchas maneras, las controversias que surgieron en torno a la supuesta supresión de noticias conservadoras o la circulación de noticias falsas durante las elecciones presidenciales de 2016 [en los Estados Unidos] pueden considerarse como el tipo de chispa que podría haber encendido una discusión de este estilo. $\mathrm{O}$, sin duda, se podría imaginar una plataforma de medios digitales como Facebook, con su posición dominante en el mercado, cada vez más preocupada por la posibilidad de que se aplique un análisis de competencia -con una lógica más orientada a los medios- por su creciente dominio tanto en el mercado económico como en el mercado de las ideas (véase Thompson, 2016). Los datos recientes muestran hasta qué punto se ha desarrollado un duopolio Facebook/Google en publicidad online y móvil (Slefo, 2017). El hecho sorprendente de que tales hallazgos no hayan desencadenado aún un debate en el discurso de las políticas públicas de los Estados Unidos tal vez pueda atribuirse al éxito que hasta ahora ha tenido la retórica de "somos una compañía tecnológica y no un medio de comunicación".

Tal como esta discusión ha intentado dejar en claro, la pregunta sobre si las plataformas como Facebook y Twitter son compañías de medios o de tecnología no es solo una cuestión de semántica sino parte de una estrategia discursiva más amplia. El éxito de esta estrategia tiene importantes consecuencias legales y de políticas públicas, y las motivaciones económicas, legales y políticas de las plataformas digitales hacen que esta (mal) interpretación de sí mismas como compañías tecnológicas en lugar de compañías de medios sea tan convincente. 


\section{4. ¿Por qué es importante?}

"Las palabras importan", tal como lo han señalado investigadores en políticas de comunicación (Lentz, 2013). Los términos específicos empleados en el discurso y en los documentos que dan forma y reflejan las decisiones de políticas públicas tienen profundas consecuencias. Por lo tanto, se utilizan estratégicamente, a menudo en un esfuerzo por definir los contornos de un problema o para influir sobre los actores [stakeholders] comprendidos dentro de los parámetros de determinada propuesta de política pública (véaase, por ejemplo, Lentz, 2011; Streeter, 1987). Una estrategia discursiva específica de varios actores [stakeholders], dentro del contexto de los medios y las comunicaciones, ha sido emplear un enfoque fuertemente tecnológico, tratando a las tecnologías digitales de las comunicaciones y los medios como agentes autónomos y/o definiendo de manera acotada el terreno de las políticas públicas exclusivamente en términos de problemas técnicos complejos, excluyendo preocupaciones sociales más amplias (Napoli, 2009; Streeter, 1987). Los académicos especializados en gobernanza de Internet, por ejemplo, han observado una tendencia entre varias partes interesadas [stakeholders] al tratar de despojar diversos aspectos de su significado político y cultural más amplio, buscando en su lugar encuadrar estas cuestiones en términos técnicos más estrechos, un proceso que en última instancia margina a las partes interesadas y a los problemas de gobernanza de un enfoque fundamentalmente político o cultural (véase, por ejemplo, Raboy y Landry, 2005). Esta tecnocratización del discurso de las políticas públicas sirve como un importante telón de fondo para el argumento "somos una compañía tecnológica y no un medio de comunicación” que aquí se considera.

En última instancia, encuadrar a las plataformas de redes sociales y a los agregadores de contenido digital exclusivamente como empresas tecnológicas margina las crecientes dimensiones políticas y culturales de su operación, que se hacen más pronunciadas a medida que estas plataformas se vuelven guardianes [gatekeepers] centrales de noticias e información en el ecosistema de medios contemporáneo. En estas situaciones, donde hay una desconexión entre la función y el encuadre, tenemos un "discurso [que] sirve para dar forma a una institución que falla en describir" (Streeter, 1987: 176). Este encuadre tecnocéntrico puede contribuir a que las plataformas operen en gran medida fuera de los marcos legales y regulatorios establecidos para las organizaciones de medios electrónicos; marcos que se establecieron en gran parte debido a las importantes dimensiones políticas y culturales de su funcionamiento. 
Por qué las empresas de medios insisten en que no son empresas de medios, por qué están equivocadas y por qué es importante

Esta situación se vuelve particularmente preocupante, ya que estas plataformas evolucionan inevitablemente de manera que invalidan aún más la distinción entre compañía tecnológica y medio de comunicación. Por ejemplo, como se señaló previamente, al igual que las generaciones anteriores de distribuidores de contenidos, como los sistemas de cable y satélite y, más recientemente, YouTube y Netflix (Kokalitcheva, 2016; Napoli, 2016), Facebook ha comenzado a integrarse verticalmente con la creación de contenidos. La compañía ha iniciado un esfuerzo para producir programación de video original (Kafka, 2014). Y, a raíz de las críticas masivas sobre el papel de Facebook en la difusión de noticias falsas durante las elecciones de 2016 [en los Estados Unidos], la compañía ha iniciado el Proyecto de Periodismo de Facebook [Facebook Journalism Project]. Entre los objetivos de esta iniciativa se hallan el "forjar vínculos más profundos con los editores por medio de la colaboración con herramientas y características de publicación" (Isaac, 2017: 1). Esta integración más profunda en la dinámica de la producción y distribución de noticias es, quizás, el primer paso hacia una verdadera integración vertical en el ámbito de las noticias; pero es, como mínimo, un cambio en la dirección de la organización en relación al periodismo que socava aún más los argumentos que se oponen a la categorización como una empresa de medios (Baram, 2017).

Tal vez no sea sorprendente, entonces, que el CEO de Facebook, Mark Zuckerberg, haya comenzado a suavizar ligeramente su postura con respecto a la identidad organizativa de Facebook. En diciembre de 2016 describió a Facebook "no como una compañía de medios tradicional" (en Constine, 2016, énfasis agregado). La posición evolutiva de Zuckerberg recuerda a Google, que después de años de insistir en que no era una compañía de medios declaró en su reporte anual ante la SEC [U.S. Security and Exchange Commission] en 2005 que "comenzamos como una empresa de tecnología y hemos evolucionado hacia una de software, tecnología, Internet, publicidad y medios todo en uno" (citado en BIA/Kesley, 2005). En ambos casos, la postura consiste en continuar manteniendo puntos significativos de distinción y separación de las compañías de medios tradicionales. Más allá de ello, en el momento en que estas firmas reconocen que son empresas de medios de comunicación de algún tipo, ya han cosechado varios beneficios asociados con el hecho de no ser percibidas o tratadas como compañías de medios. En efecto, parte de la forma en que estas firmas son capaces de evolucionar hacia empresas mediáticas dominantes es evadiendo los marcos legales y regulatorios aplicables a los medios de 
comunicación, los que pueden inhibir su rápida expansión y/o imponer mayores responsabilidades legales y/o costos de responsabilidad social a lo largo del camino. Y la estrategia discursiva discutida aquí probablemente juegue un papel en facilitar esta elusión.

Es importante enfatizar que no hemos buscado aquí elaborar recomendaciones para realizar intervenciones legales o regulatorias específicas. Nuestro objetivo simplemente ha sido resaltar los defectos lógicos y las motivaciones subyacentes de una estrategia discursiva que desvincula a las plataformas de redes sociales de los marcos normativos, legales y regulatorios que tradicionalmente han sido aplicados a aquellas instituciones que desempeñaron las funciones que estas plataformas tienen ahora. Por este motivo, hemos intentado rechazar esta caracterización puramente tecnológica de las redes sociales y los agregadores de contenido digital, con la esperanza de que el discurso de la política pública y la gobernanza sobre estas plataformas refleje mejor el hecho de que los puntos de continuidad entre los medios tradicionales y los nuevos son más fuertes de lo que se sugiere.

\section{Conclusión}

A lo largo de la historia de los medios, las tecnologías y los servicios de comunicación han evolucionado para cumplir funciones que, finalmente, son muy diferentes de aquellas para las que fueron creados. La radio fue creada para facilitar la comunicación entre los barcos y la tierra firme. La videograbadora se creó para facilitar la grabación de videos domésticos, ya que sus inventores no tenían idea acerca de la enorme industria de venta/ alquiler de videos que surgiría. Internet fue creada para facilitar la comunicación entre el gobierno y los investigadores académicos. Facebook fue creado para ayudar a los estudiantes universitarios a identificar compañeros atractivos.

La velocidad con la que pueden evolucionar las funcionalidades de las tecnologías de la comunicación puede llevar a un estado perceptivo de retraso persistente con respecto a la realidad. Cuando la funcionalidad en cuestión implica informar e influir sobre el proceso democrático, dicho retraso perceptivo puede tener ramificaciones peligrosas y profundas. Las elecciones de 2016 [en los Estados Unidos] parecen haber provocado cierta actualización en este sentido, ya que los usuarios, periodistas, responsables políticos, académicos, e incluso los 
Por qué las empresas de medios insisten en que no son empresas de medios, por qué están equivocadas y por qué es importante

ejecutivos de estas plataformas, se encuentran desarrollando un sentido más claro del papel cada vez más influyente que éstas se hallan jugando en el proceso democrático. Aún está por verse si esta mayor claridad conduce a normas y estructuras de gobierno que sirvan mejor al interés público, algo que debería ser un foco de investigación a futuro.

Finalmente, por mucho que este artículo haya sostenido que las plataformas de redes sociales y los agregadores de contenido digital encajan bastante bien dentro de los parámetros de las organizaciones de medios, se podría argumentar con toda facilidad que es necesario y apropiado que nuestra comprensión de las empresas de medios evolucione completamente para abarcar la estructura y el funcionamiento de estas plataformas. Las aproximaciones conceptuales recientes sobre los medios de comunicación parecen reflejar esta perspectiva (véase Hess, 2014). Deuze (2007: 9), por ejemplo, enfatiza que "las industrias de medios producen contenido, sí, pero también invierten en plataformas para la conectividad". Quizás sea hora de delinear de manera más rigurosa, como mínimo, los contornos y los parámetros de la contemporánea e híbrida empresa de tecnología/medios, y comenzar a articular si estas compañías deberían encajar en los marcos legales, regulatorios y periodísticos existentes y de qué modo; o si es preciso idear nuevos encuadres o modificar los existentes para que reflejen su naturaleza heterogénea.

\section{Referencias}

Baram, M. (2017, 11 de enero). Facebook finally admits it's a giant media company - almost. Fast Company. Recuperado de https://www.fastcompany.com/3067148/facebook-finally-admits-its-agiant-media-company-almost

Bell, E. (2016, 7 de marzo). Facebook is eating the world. Columbia Journalism Review. Recuperado de http://www.cjr.org/analysis/facebook and media.php

Benton, J. (2014, 11 de Agosto). Elizabeth Spiers on Buzzfeed and other 'tech' companies: "You're still a media company'. Nieman Lab. Recuperado de 
http://www.niemanlab.org/2014/08/elizabeth-spiers-on-buzzfeed-andother-tech-companies-youre-still-a-media-company/

Bhattacharyya, S. (2016, 26 de octubre). Digital ads to overtake traditional ads in U.S. local markets by 2018. Advertising Age. Recuperado de http://adage.com/article/cmo-strategy/local-ads-digital-2018-biakelsey/306468/

BIA/Kelsey (2005, 4 de abril). Google 10-K: 'We're a media company,' and other tidbits. BIA Advisory Services. Recuperado de http://blog.biakelsey.com/index.php/2005/04/04/google-10-k-were-amedia-company-and-other-tidbits /

Bilton, N. (2012, 25 de julio). Is Twitter a media company or a technology company?. New York Times. Recuperado de http://bits.blogs.nytimes.com/2012/07/25/is-twitter-a-media-ortechnology-company/

Bogust, I. (2016, 3 de Agosto). Facebook is not a technology company. Atlantic. Recuperado de http://www.theatlantic.com/technology/archive/2016/08/facebook-isnot-a-technology-company/494183/

Bond, P. (2007, 28 de septiembre). Fresh Facebook draws attention. Hollywood Reporter. Recuperado de http://www.hollywoodreporter.com/news/freshfacebook-draws-attention-151237

Caplan, R. (2016, 17 de mayo). Like it or not, Facebook is now a media company. New York Times. Recuperado de http://www.nytimes.com/roomfordebate/2016/05/17/is-facebooksaving-journalism-or-ruining-it/like-it-or-not-facebook-is-now-a-mediacompany

Carney, M. (2015, 20 de febrero). As Uber fights to maintain its technology company classification in India, the rest of the world watches. Pando. Recuperado de https://pando.com/2015/02/20/as-uber-fights-tomaintain-its-technology-company-classification-in-india-the-rest-of-theworld-watches/ 
Por qué las empresas de medios insisten en que no son empresas de medios, por qué están equivocadas y por qué es importante

Constine, J. (2016, 21 de diciembre). Zuckerberg implies Facebook is a media company, just 'not a traditional media company' TechCrunch. Recuperado de https://techcrunch.com/2016/12/21/fbonc

Constine, J. (2015, 12 de mayo). Facebook starts hosting publishers' 'instant articles'. TechCrunch. Recuperado de https://techcrunch.com/2015/05/12/facebook-instant-articles/

Cukier, J. (2016, 1 de marzo). Why is Uber considered a technology company and a lot of people place it among tech giants like Google, Facebook, etc?. Quora . Recuperado de https://www.quora.com/Why-is-Uber-considereda-technology-company-and-a-lot-of-people-place-it-among-tech-giantslike-Google-Facebook-etc

Curtin, M.; Holt, J y Sanson, K (eds.) (2014). Distribution revolution: Conversations about the digital future of film and television. Berkeley: University of California Press.

Deuze, M. (2007). Media work. Cambridge: Polity Press.

DeVito, M. (2016). From editors to algorithms: A values-based approach to understanding story selection in the Facebook news feed. Digital Journalism 5 (6), 1-21. DOI http://dx.doi.org/10.1080/21670811.2016.1178592

Dixon, C. (2014, 10 de agosto). BuzzFeed. CDixon Blog. Recuperado de http://cdixon.org/2014/08/10/buzzfeed/

D'Onfro, J. (2016, 29 de agosto). Facebook is telling the world it's not a media company, but it might be too late. Business Insider. Recuperado de http://www.businessinsider.com/mark-zuckerberg-on-facebook-being-amedia-company-2016-8

Dougherty, C y Steel, E. (2015, 21 de octubre). YouTube introduces YouTube Red, a subscription service. New York Times. Recuperado de https://www.nytimes.com/2015/10/22/technology/youtube-introducesyoutube-red-a-subscription-service.html

Etherington, D. (2016, 14 de diciembre). Facebook exploring creation of its own content. TechCrunch. Recuperado de 
https://techcrunch.com/2016/12/14/facebook-exploring-creation-of-itsown-original-video-content/

Fiegerman, S. (2016, 15 de junio). Dear Facebook, you're a media company now. Start acting like one. Mashable. Recuperado de http://mashable.com/2016/05/15/facebook-media-company/

Fiveash, K. (2016, 20 de agosto). 'We're a tech company not a media company,' says Facebook founder. Ars Technica. Recuperado de https://arstechnica.com/tech-policy/2016/08/germany-facebook-edithateful-posts-zuckerberg-says-not-media-empire/

Foremski, T. (2004, 11 de diciembre). Stuff you didn't know about Google — A report from the Googleplex Xmas party. SiliconV alleyWatcher. Recuperado de

http://www.siliconvalleywatcher.com/mediawatch/archives/2004/12/stu ff you didnt.php

Fox, J. (2014, 13 de agosto). Why it's good to be a 'technology company'. Harvard Business Review. Recuperado de https://hbr.org/2014/08/why-its-good-tobe-a-technology-company/

Gillespie, T. (en prensa). Regulation of and by platforms. En Burgess, J., Poell, T. y Marwick, A. (eds). Sage bandbook of social media. Thousand Oaks, Calif.: SAGE. Recuperado de https://www.microsoft.com/en-us/research/wpcontent/uploads/2016/12/Gillespie-Regulation-ofby-PlatformsPREPRINT.pdf

Gillespie, T. (2017). Algorithmically recognizable: Santorum's Google problem and Google's Santorum problem. Information, Communication \& Society, 20 (1), 63-80. DOI http://dx.doi.org/10.1080/1369118X.2016.1199721

Gillespie, T. (2010). The politics of 'platforms'. New Media \& Society, 12 (3). $347-$ 364. DOI http://dx.doi.org/10.1177/1461444809342738,

Gottfried, J. y Shearer, E. (2016, 26 de mayo). News use across social media platforms 2016. Pew Research Center. Recuperado de http://www.journalism.org/2016/05/26/news-use-across-social-mediaplatforms-2016/ 
Por qué las empresas de medios insisten en que no son empresas de medios, por qué están equivocadas y por qué es importante

Guynn, J. (2016, 16 de noviembre). Twitter suspends alt right accounts. USA Today. Recuperado de http://www.usatoday.com/story/tech/news/2016/11/15/twittersuspends-alt-right -accounts/93943194/

Helft, M. (2008, 10 de agosto). Is Google a media company?. New York Times. Recuperado de http://www.nytimes.com/2008/08/11/technology/11google.html

Hess, T. (2014). What is a media company? A reconceptualization for the online world. International Journal on Media Management 16 (1). 3-8. DOI http://dx.doi.org/10.1080/14241277.2014.906993

Ingram, M. (2012, 16 de mayo). Facebook's biggest problem is that it's a media company. Gigaom. Recuperado de https://gigaom.com/2012/05/16/facebooks-biggest-problem-is-that-itsa-media-company/

Isaac, M. (2017, 11 de enero). Facebook, nodding to its role in media, starts a journalism project. New York Times. Recuperado de https://www.nytimes.com/2017/01/11/technology/facebook-journalismproject.html

Isaac, M. (2016, 12 de mayo). Facebook, facing bias claims, shows how editors and algorithms guide news. New York Times. Recuperado de http://www.nytimes.com/2016/05/13/technology/facebook-guidelinestrending-topics.html

Jarvis, J. (2016, 11 de mayo). Facebook needs an editor. Medium. Recuperado de https://medium.com/whither-news/facebook-needs-an-editor$\underline{\mathrm{b} 388 \mathrm{f} 789 \mathrm{a} 9 \mathrm{c} 3}$

Kafka, P. (2014, 11 de agosto). How Jonah Peretti and BuzzFeed got to 850 million. Recode. Recuperado de http:/ /www.recode.net/2014/8/11/11629746/how-jonah-peretti-andbuzzfeed-got-to-850-million. 
Kitchin, R. (2017). Thinking critically about and researching algorithms. Information, Communication \& Society 20 (1). 14-29. DOI http://dx.doi.org/10.1080/1369118X.2016.1154087.

Koetsier, J. (2012, 22 de agosto). Exclusive: Cheezburger will take page out of Reddit's playbook, allow users to create own subsites. Venturebeat. Recuperado de http://venturebeat.com/2012/08/22/exclusivecheezburger-will-take-page-out-of-reddits-playbook-allow-users-to-createown-subsites/

Kokalitcheva, K. (2016, 10 de febrero). YouTube's first lineup of original content is here. Fortune. Recuperado de http://fortune.com/2016/02/10/youtube-originals-release/

Kramer, S. D. (2006, 12 de junio). Google is still a tech company. Really. Gigaom. Recuperado de https://gigaom.com/2006/06/12/google-is-still-a-techcompany-really/

Langer, A. (2003, 10 de septiembre). Is Steve Jobs the god of music? Esquire. Recuperado de http://www.esquire.com/news-politics/a11177/steve-jobsesquire-interview-0703/

Lentz, R. (2013). Excavating historicity in the U.S. network neutrality debate: An interpretive perspective on policy change. Communication, Culture, \& Critique 6 (5). 568-597. DOI http://dx.doi.org/10.1111/cccr.12033

Lentz, R. (2011). Regulation as linguistic engineering. En Mansell, R. y Raboy, M. (eds). Handbook of global media and communication policy. New York: Blackwell 432-448. DOI http://dx.doi.org/10.1002/9781444395433.ch27

Manjoo, F. (2016, 11 de mayo). Facebook's bias is built-in, and bears watching. New York Times. Recuperado de http://www.nytimes.com/2016/05/12/technology/facebooks-bias-isbuilt-in-and-bears-watching.html,

Gunther McGrath, R. (2015, 10 de enero). 15 years later, lessons from the failed AOL-Time Warner merger. Fortune. Recuperado de http://fortune.com/2015/01/10/15-years-later-lessons-from-the-failedaol-time-warner-merger/ 
Por qué las empresas de medios insisten en que no son empresas de medios, por qué están equivocadas y por qué es importante

Mickey, B. (2013, 29 de octubre). Are you a technology company or a media company? Folio. Recuperado de http://www.foliomag.com/are-youtechnology-company-or-media-company/

Morrissey, B. (2016, 6 de mayo). BuzzFeed's Dao Nguyen: 'We don't think of ourselves as an island'. Digiday. Recuperado de http://digiday.com/publishers/buzzfeed-dao-nguyen-digiday-podcast/

Nadler, A. M. (2016). Making the news popular: Mobilizing U.S. news audiences. Champaign: University of Illinois Press.

Napoli, P. M. (2016). Requiem for the long tail: Towards a political economy of content aggregation and fragmentation. International Journal of Media \& $\begin{array}{lllll}\text { Cultural } & \text { Politics } & 12 & \text { (3). } & 341-356 .\end{array}$ DOI http://dx.doi.org/10.1386/macp.12.3.341_1.

Napoli, P. N. (2015). Social media and the public interest: Governance of news platforms in the realm of individual and algorithmic gatekeepers.

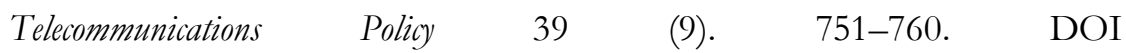
http://doi.org/10.1016/j.telpol.2014.12.003

Napoli, P. N. (2011). Audience evolution: New technologies and the transformation of media audiences. New York: Columbia University Press.

Napoli, P. N. (2008). Bridging cultural policy and media policy. Journal of Arts Management, Law, and Society 37 (4). 311-322. DOI http://dx.doi.org/10.3200/JAML.47.4.311-332

Napoli, P. N. (2001). Foundations of communications policy: Principles and process in the regulation of electronic media. Cresskill, NJ: Hampton Press.

Napoli, P. N. y Caplan, R. (2016). When media companies insist they're not media companies and why it matters for communications policy. Ponencia presentada en la Telecommunications Policy Research Conference (Arlington, Va.). Recuperado de http://dukespace.lib.duke.edu/dspace/handle/10161/13897.

New York v. Harris, 2012. 36 Misc. 3d 868; 949 N.Y.S.2d 590; 2012 N.Y. Misc. LEXIS 3076; 2012 NY Slip Op 22175. Ver también https://www.eff.org/cases/new-york-v-harris. 
Nunez, M. (2016a, 9 de mayo). Former Facebook workers: We routinely suppressed conservative news. Gizmodo. Recuperado de http://gizmodo.com/former-facebook-workers-we-routinely-suppressedconser-1775461006,

Nunez, M. (2016b, 3 de mayo). Want to know what Facebook really thinks of journalists? Here's what happened when it hired some. Gizmodo. Recuperado de http://gizmodo.com/want-to-know-what-facebook-really-thinks-ofjournalists-1773916117

Pasquale, F. (2015). The black box society: The secret algorithms that control money and information. Cambridge, Mass.: Harvard University Press.

Patel, N. (2014, 15 de enero). The wrong words: How the FCC lost net neutrality and could kill the Internet. The Verge. Recuperado de http://www.theverge.com/2014/1/15/5311948/net-neutrality-and-thedeath-of-the-internet

Picard, R. (2011). The economics and financing of media companies. Segunda edición. New York: Fordham University Press.

Perez, S. y Shieber, J. (2017, 27 de febrero). YouTube Unveils YouTube TV, its live TV streaming service. TechCrunch. Recuperado de https://techcrunch.com/2017/02/28/youtube-launches-youtube-tv-itslive-tv-streaming-service/

Pierson, D. y Dave, P. (2016, 31 de mayo). If Facebook promotes propaganda, can it be a neutral news platform? Los Angeles Times. Recuperado de http://www.latimes.com/business/la-fi-tn-eu-tech-20160531-snapstory.html

Raboy, M. y Landry, N. (2005). Civil society, communication, and global governance: Issues from the World Summit on the Information Society. New York: Peter Lang.

Rana, S. (2012, 12 de julio). Is Twitter a technology platform, a media company - or both? Tech2. Recuperado de http://tech.firstpost.com/newsanalysis/is-twitter-a-technology-platform-a-media-company-or-both29998.html 
Por qué las empresas de medios insisten en que no son empresas de medios, por qué están equivocadas y por qué es importante

Rinehart, W. (2015). A semantic network analysis of the network neutrality debate. Ponencia presentada en la Research Conference on Communication, Information and Internet Policy. Recuperado de https://tprc2015.sched.com/event/3v9Q/a-semantic-network-analysis-ofthe-network-neutrality-debate

Robinson, J. (2016, 23 de noviembre). Merkel vows to regulate 'fake news' after blaming social media for the rise of populist politics and 'making it harder to form people's opinions than it was 25 years ago'. Daily Mail. Recuperado de http://www.dailymail.co.uk/news/article-3964366/Merkel-vowsregulate-fake-news-blaming-social-media-rise-populist-politics-makingharder-form-people-s-opinions-25-years-ago.html

Roberts, J. J. (2016, 14 de noviembre). Why Facebook won't admit it's a media company. Fortune. Recuperado de http://fortune.com/2016/11/14/facebook-zuckerberg-media/

Ruane, K. A. (2011, 13 de julio). Fairness Doctrine: History and constitutional issues. Congressional Research Service. Recuperado de https://www.fas.org/sgp/crs/misc/R40009.pdf 